\title{
Role of Veterinarians and Livestock Owners in Achieving One Calf Per Year in Dairy Cows
}

\section{Swati Ruhil*}

Scientist, Haryana Pashu Vigyan Kendra, Lala Lajpat Rai University of Veterinary and Animal Sciences, Uchani, Karnal, Haryana, India

*Corresponding Author: Swati Ruhil, Scientist, Haryana Pashu Vigyan Kendra, Lala Lajpat Rai University of Veterinary and Animal Sciences, Uchani, Karnal, Haryana, India.

Obtaining a calf every year in a dairy herd appears to be a difficult goal yet is achievable with concerted efforts of dairy farmers and veterinarians. The core role of dairy veterinarians remains individual animal examinations but this must be supplemented with systematic herd fertility investigation and veterinarian-led herd fertility management. This new role encompasses leading the change from clinical calls only to a planned approach to herd fertility, demonstrating the cost-benefits of the program, scheduling fertility management consultations, assisting the farmer in setting specific, measurable, attainable, relevant and time-limited goals. The application of such goals in dairy herds is possible when the dairy farmer is ready to adopt the newer protocols of fixing voluntary waiting periods and applying estrus synchronization and timed inseminations with high fertility semen. For individual cows the role of the owner is increased as compared to the veterinary professional. An important consideration for the farmer is not to wait for prolonged periods of 6 months to get his cows pregnant again post calving. It is the prime duty of the veterinary professionals to educate the farmer about 2 important things- 1) Prompt examination of animals not returning to estrus within 70 days of calving and adopting necessary therapy and 2) Necessary examination of animals 60-90 days post insemination for pregnancy and therapy of non-pregnant animals. These 2 important parameters would help the farmers in attaining one calf a year. The importance of early resumption of post-partum estrus has been stressed in many studies but the pivotal role appears to be the management of cows during the immediate post-partum period and minimizing the negative energy balance during this period. Metabolic shifts during the post-partum period can be minimized by feeding cows during pregnancy period with low energy high fiber and supplementing pregnant cows with $20 \%$ of post -partum concentrate one
Received: July 02, 2021

Published: August 01, 2021

(C) All rights are reserved by Swati Ruhil.

\section{Volume 3 Issue 9 September 2021 (C) All rights are reserved by Swati Ruhil.}

month ahead of parturition. Post-partum complications involving the reproductive tract can be minimized by observation of proper parturient hygiene. Thus, concerted efforts of the dairy farmers and veterinarians are needed in achieving the goal of one calf a year for maintaining the profitability of the dairy herd. 